\section{REFLECTING ALL YOUR MIRROR NEEDS}

Technimirror has been designing and producing high quality, double-sided dental photography mirrors for dental hygiene for the past five years.

Each Technimirror product is carefully inspected at every stage from selection of the raw materials until the final polishing stage. Based in France, Technimirror sells internationally, direct from their factory to your lab. All Technimirror products are securely packaged to ensure safe delivery.

Technimirror offers two types of specialised dental photography mirrors: the dental mirror Chromium collection has high reflectivity, up to $70 \%$, and the dental mirror HR (Titanium) collection has very high reflectivity, close to $80 \%$. Both mirrors have double-sided coating, no ghost picture and offer excellent value for money and discounts when ordered in bulk. Titanium mirrors also have high durability to autoclaving and are resistant to scratches.

Technimirror also offers an option to create your own customised mirror designed and manufactured to your requirements. All products are made in France, are ultra long-life, ergonomic, and have a state-of-the-art coating. Reader response number 53

\section{MONITOR YOUR LEGIONELLA CONTROL}

It has only recently come to light that the water services and the associated dental equipment for many dental surgeries have escaped the important attention of Legionella Control in the UK. A selection of findings from available studies show why these controls should be put in place:

- Legionella pneumophila was detected in $86.7 \%$ of the dental units at the beginning of the working day

- Thirty-three percent of 34 dental surgeries had Legionella bacteria

- Experts stress that the microbial presence in dental unit water may be a real threat to medically compromised patients (the elderly, immunesuppressed, and HIV-positive patients).
Chapter 19 of the HTM 01-05 memorandum devotes itself primarily to Legionella and states:

- Risk Assess (19.2)

- Provide control measures (19.3)

- Monitor your control measures, define those responsible, and ensure adequate training is carried out (19.3).

In an age where inadequate infection control and prevention in healthcare facilities is seldom absent from the media, it is vital that dental healthcare professionals have the relevant controls in place.

eplus Global are a specialist in the Water Services Industry and have over 50 years of experience within the company. Reader response number 54

\section{PNEUMATIC SADDLE SEATS}

Meditelle Dental are introducing exciting new options on their popular dental seating models.

Medi Saddles with back and torso supports will be available with a pneumatic foot height control, offered previously on the standard Medi Stool. In response to requests from dental surgeons the pneumatic foot control with hands free height adjustment will also be offered on the Tri-seating range.

Meditelle Dental, who manufacture bespoke specialist seating in their
Birmingham UK factory, also offer a leather hide covering option on all saddle seating.

Reader response number 55

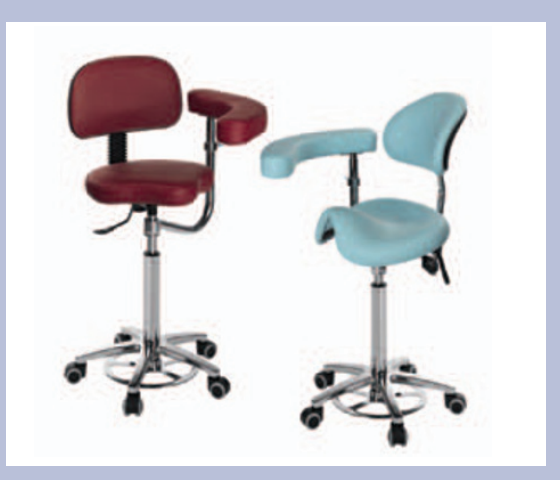

